\title{
Strong effect of magnetic field on the edge luminescence line width in diluted magnetic narrow-gap $\mathrm{Hg}_{1-\mathrm{x}} \mathrm{Mn}_{\mathrm{x}} \mathrm{Te}$
}

\author{
Yu. I. Mazur, \\ Institute of Semiconductor Physics, NAS Ukraine, 45 prospekt Nauki, Kyiv, 252028, Ukraine
}

\begin{abstract}
Peculiarities of photoluminescence in narrow-gap $\mathrm{Hg}_{1-\mathrm{Mn}} \mathrm{Me}(0.09<x<0.11)$ under variation of both magnetic field and temperature are studied. A strong resonance enhancement of the edge emission and a narrowing of the line width to less than $2.5 \mathrm{meV}$ were detected. The photoluminescence behaviour observed can be well described by the model of a magnetically trapped exciton.
\end{abstract}

Keywords: Infrared luminescence, $\mathrm{Hg}_{1-\mathrm{x}} \mathrm{Mn}_{\mathrm{x}} \mathrm{Te}$, exciton magnetic polaron.

Paper received 02.06.98; revised manuscript received 27.07.98; accepted for publication 27.10.98.

\section{Introduction}

Electronic properties of narrow-gap semiconductors are strongly modified by application of the magnetic field, resulting in localization of electrons in fluctuation potential wells of impurities [1], formation of a viscous electron fluid or Wigner crystallization [2], and magnetic freeze-out of electrons on individual donors [3]. The results can be quite different depending on whether the semiconductor is compensated or not. Specific peculiarities are caused by magnetic ions, such as $\mathrm{Mn}^{2+}$, incorporated into the crystal lattice, due to the exchange coupling between their localized magnetic moments and the spins of free charge carriers. In the presence of the external magnetic field, the energies of Landau sublevels include the exchange contribution related to d-electron magnetization and become strongly temperature-dependent. Also, impurity states show the effect of exchange interaction. Acceptors produced by mercury vacancies or foreign impurities in $\mathrm{Hg}_{1-\mathrm{x}} \mathrm{Mn}_{\mathrm{x}} \mathrm{Te}$ demonstrate the boiloff effect in the ultra-quantum limit [4]. More complex systems, such as excitons bound to acceptors and a bound magnetic polaron $[5,6]$ are widely involved when results of magnetooptical studies are interpreted. A particular set of experiments provides data on the flare-up of discrete features in absorption or emission from narrow-gap semiconductors when the magnetic field is applied [7-11]. In fact, these data confirm that application of the magnetic field of appropriate magnitude can stabilize the electronic states even in the case of significant disordering in semiconductors. Here we present new evidence for such magnetic field induced stabilization based on the strong narrowing of infrared luminescence observed in diluted small-gap $\mathrm{Hg}_{1-x} \mathrm{Mn}_{\mathrm{x}} \mathrm{Te}$.

\section{Magnetoluminescence in $\mathrm{Hg}_{1-\mathrm{x}} \mathrm{Mn}_{\mathrm{x}}$ Te single crystals}

A set of eight $\mathrm{Hg}_{1-x} \mathrm{Mn}_{\mathrm{x}} \mathrm{Te}$ single crystals $(0.09<x<0.11)$ grown by the traveling heater technique was investigated. The molar fraction values for all samples corresponded to the energy gap $\left(E_{g}\right)$ smaller than $140 \mathrm{meV}$. Magnetoluminescence measurements in the wavelength range from 8 to $14 \mathrm{~mm}$ were carried out at excitation densities below $1 \mathrm{~W} / \mathrm{cm}$. This was possible due to a new experimental system consisting of a Spectromag 4000 magnet (7 T) and an infrared Fourier-transform spectrometer IFS 88 . The double modulation technique was employed for phase-locked PL excitation and detection. The magnetic field $(B)$ and temperature $(T)$ were varied within the range $0 \leq \mathrm{B} \leq 7 \mathrm{~T}$ and $5 \leq T \leq 140 \mathrm{~K}$.

Typically, all spectra consist of two features separated by the distance of about $15 \mathrm{meV}$, interpreted as edge emission (the high-energy feature) and impurity related (the lowenergy one). At low temperatures ( $5 \mathrm{~K}$ ) in the absence of the magnetic field, only a poor PL signal was detected. An increase of the magnetic field and, surprisingly, temperature substantially enhances the edge emission line. Fig. 1 shows PL spectra for various values of the magnetic field at $T=5 \mathrm{~K}$ for compounds with the molar fraction $x=0.095$ (fig. 1(a)) and $x=0.104$ (fig. 1(b)). These spectra were detected in the Voight geometry $(\vec{E} \mid \vec{B})$. The magnetic field induced enhancement of the edge emission line is accompanied by the extreme line narrowing down to the full width at half maximum (FWHM) of less than $2.5 \mathrm{meV}$. To the best of our knowledge, this is the smallest FWHM ever reported for spontaneous emission from narrow-gap semiconductors. 
Yu. I. Masur: Strong effect of magnetic field on the edge luminescence line...
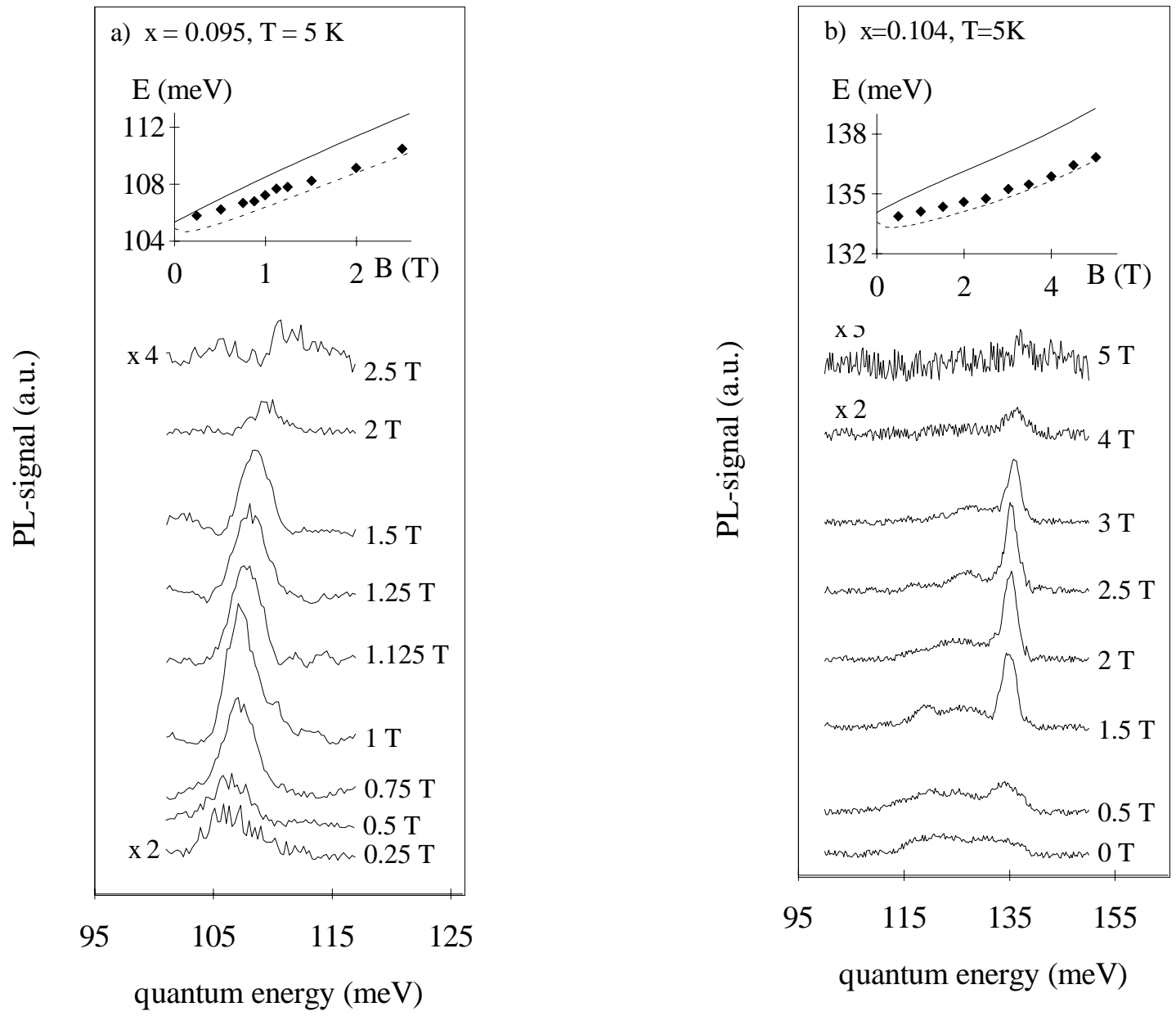

Fig. 1. Magnetic tield-1nquced photoluminescence of narrow-gap $\mathrm{Hg}_{1-\mathrm{x}} \mathrm{Mn}_{\mathrm{x}} \mathrm{Te}$ single crystals: (a) $x=0.095$; (b) $x=0.104 . T=5 \mathrm{~K}$, Voight geometry. Insets: PL peak positions versus magnetic field, points - experiment, solid curves - energies of $b_{\Gamma_{8}}(0) \rightarrow a_{\Gamma_{6}}(-1)$ transitions calculated for a modified Pidgeon-Brown model with (dashed curve) and without excitonic corrections.

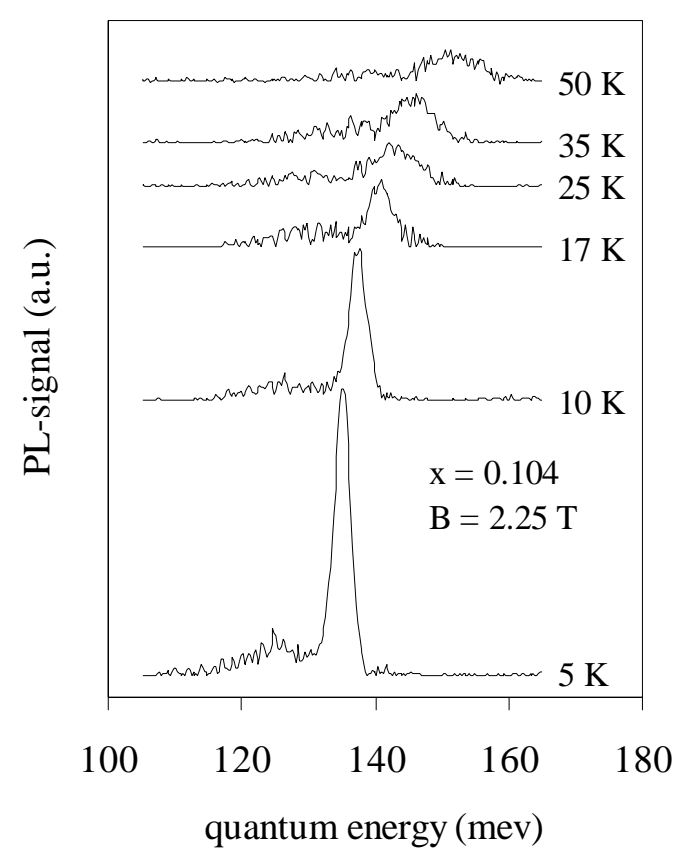

After passing the signal maximum (and the FWHM minimum) at $B_{\max } \cong 1.1 \mathrm{~T}$ for $x=0.095$ and $B_{\max } \cong 2.25 \mathrm{~T}$ for $x=$ $=0.104$, the PL signal decreases again and vanishes in the noise at about $3 \mathrm{~T}$ and $5 \mathrm{~T}$, respectively.

The temperature dependence of the PL signal in the absence of the magnetic field also exhibits a non-monotonic behavior with a maximum at $T_{\max }=35 \mathrm{~K}$ for $x=0.104$ and $T_{\max }=90 \mathrm{~K}$ for $x=0.095$. Quenching of the PL signal at a constant magnetic field is demonstrated in fig. 2. These measurements were carried out in the Faraday geometry $(\vec{E} \perp \vec{B})$. The strong signal of spontaneous emission observed at $B=$ $=B_{\max }$ and a low temperature $T=5 \mathrm{~K}$ is rapidly quenched when the temperature increases. Fig. 2 demonstrates the transformation of PL spectra with temperature for the molar fraction $x=0.104$ and magnetic field $B=2.25 \mathrm{~T}$. The behavior of the PL feature observed is the subject of our primary interest.

Fig. 2. Temperature evolution of the PL feature: $x=0.104 ; B=$ $=B_{\max }=2.25 \mathrm{~T}$, Faraday geometry. 


\section{Yu. I. Masur: Strong effect of magnetic field on the edge luminescence line...}

\section{Theory}

In the following section, we will discuss the temperature and magnetic field dependences of the short-wavelength PL line, which is edge-related. The magnetic field behavior of this line has a similarity to that of the strongest spectral features of PL reported by Gel'mont et al. [8] for $\mathrm{Hg}_{1-\mathrm{x}} \mathrm{Mn}_{\mathrm{x}} \mathrm{Te}(0.12 \leq x \leq 0.13)$. The latter feature was ascribed to the conduction band to acceptor level recombination. Its strong non-monotonic dependence on the magnetic field has been interpreted in terms of the recombination probability which was assumed to be proportional to the effective volume of the hole. For $B=0 \mathrm{~T}$ the effective volume $V_{\text {eff }} \sim a_{h}^{3}$, where $a_{h}$ is the Bohr radius of heavy holes. With the magnetic field, $a_{h}$ increases significantly, and $V_{\text {eff }}$ grows, resulting in an enhancement of the PL intensity. $B$-induced enlargement of the Bohr radius reflects the magnetic fieldinduced anisotropy of the hole (and acceptor) wave function, which gradually decreases at stronger magnetic fields. This leads to a smaller probability of recombination and, consequently, to luminescence fading. Formerly we have accepted this mechanism as consistent with the PL behavior for our case. However, our recent comprehensive investigations of PL for the above-mentioned molar fraction range gave new results that could hardly be understood in the framework of the former model. There is a dramatic $B$-field induced narrowing of the PL line and its high sensitivity to the magnetic field variation. Furthermore, an unusual temperature dependence should be mentioned. Starting from this point, we subsequently take into account different mechanisms. In the following consideration, we used a modified Pidgeon-Brown model to calculate the energy band structure of $\mathrm{Hg}_{1-\mathrm{x}} \mathrm{Mn}_{\mathrm{x}} \mathrm{Te}$ in the magnetic field. The Hamiltonian of $\vec{k} \vec{p}$ interaction taking into account the magnetic field is written in the form of an $8 \times 8$ matrix [13]. The exchange interaction between the conduction electrons and $3 \mathrm{~d}$ electrons of manganese ions is introduced by a simple Heisenberg-like formula:

$$
H_{s d}=\sum_{j} J\left(\vec{r}-\vec{R}_{j}\right)\left(\hat{s} \cdot \hat{S}_{j}\right)
$$

where $\hat{S}$ and $\hat{S}_{j}$ denote the spin operators of the band electron and $j$-th $\mathrm{Mn}^{2+}$ ion, respectively, and $J\left(\vec{r}-\vec{R}_{j}\right)$ is an appropriate exchange constant. The matrix elements of $H_{s d}$, calculated using the Luttinger-Kohn basis, then are reduced to two nearly diagonal $4 \times 4$ blocks $D_{a}^{\prime}$ and $D_{b}{ }^{\prime}$ [14] and added to the matrix of $\vec{k} \vec{p}$ Hamiltonian. Being solved numerically in terms of harmonic oscillator wave functions, the resultant matrix provides the $a$ and $b$ ladders (corresponding to spin directed up and down, respectively) of Landau levels both for conduction and valence bands and the wave functions corresponding to every energy state calculated.

To take into account the electron-hole interaction producing exciton states, we note that this coupling does not mix the basis functions in the effective mass approximation. Therefore, diamagnetic excitons are considered as formed by an electron from the conduction subbands and a heavy hole from the exchange split valence subbands. Here the electron-hole spin-spin interaction has to be neglected. The binding energy $E_{b i n}$ for a diamagnetic exciton is calculated from a simple analytical formula derived in [15]

$$
\begin{aligned}
& E_{b i n}=\gamma+1+2 \exp (2 / \gamma) E i(-2 / \gamma) \times \\
& \times[1+2 / \gamma \exp (2 / \gamma) E i(-2 / \gamma)]^{-1}
\end{aligned}
$$

with $\gamma=e B\left(2 \mu c R y_{0}\right)^{-1}$ and $E i(-x)$ being the exponential integral; $m$ and $R y_{0}$ are the reduced mass and exciton Rydberg. Equation (2) gives surprisingly good results in spite of a very approximate nature.

As the edge emission is discussed, band tails have to be taken into account. For narrow-gap semiconductors, the magnetic field induced changes in the band tails have been considered in details in [16-18]. New aspects arise when the magnetopolaron effect is involved. This effect includes a self-consistent decrease of the electron energy due to the electron-induced polarization of spins of localized magnetic ions. To describe the magnetic polaron, Hamiltonian (1) is usually chosen with (-) sign. The kinetic term $p^{2} / 2 m^{*}$ is to be added in eq. (1) for free magnetic polarons (FMP), and the Coulomb potential has to be introduced when bound magnetic polarons (BMP) are considered. In fact, FMP's are poorly detectable in semimagnetics, while more favorable conditions exist for the BMP observation.

Nevertheless, even in this case the donor-related BMP reveals only moderate spin correlations at $2 \mathrm{~K}$ and the coupling constant

$$
W_{0}=\left(S(S+1) / 3 \sum_{j} J_{j}^{2}\right)^{1 / 2}
$$

is typically about $0.7 \mathrm{meV}$ in wide-gap semimagnetic crystals. The most probable effect is an acceptor-related polaron. In the case of acceptor-bound magnetic polaron, two modes are probable: either a fluctuation-dominated mode at high temperatures or a saturated mode if the temperature is low enough. The coupling constant for the acceptor-related BMP is substantially larger and amounts to $W_{0} \approx 6 \mathrm{meV}$, compared to the donor BMP. Optical experiments have given proofs of the BMP existence in wide-gap semimagnetic semiconductors.

For narrow-gap semimagnetics, this problem is open. Nevertheless, this effect can also be expected under specific conditions. Here, the magnetopolaron effect can cause pronounced changes in the structure of the band tails [19]. It is known that the fluctuation potential generated by compositional disorder leads to the band edge smearing in solid solutions described in [20] by

$\ln (g(E) / g(0))=-\left(E / E_{0}\right)^{1 / 3}$

where the density of states $g(E)$ decreases exponentially for energies below $E_{g}$. The tailing energy $E_{0}$ is defined as follows

$$
E_{0}=\left(\alpha^{4} x^{2}(1-x)^{2} m_{c}^{3}\right) /\left(178 \hbar^{6} N^{2}\right)
$$


Yu. I. Masur: Strong effect of magnetic field on the edge luminescence line...

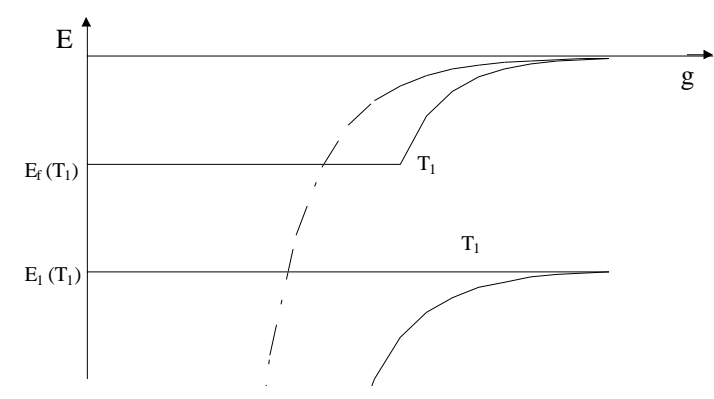

Fig. 3. A gap in the density of states function $g(E)$ caused by exchange interaction for diluted semimagnetics [19]. The case of non-correlated disorder. Dashed line shows $g(E)$ given by eq. (3).

here $\mathrm{x}$ is the averaged mole fraction and $m_{c}$ is the effective charge carrier mass, $N$ is the concentration of lattice sites containing either $\mathrm{Hg}$ or $\mathrm{Mn}$ atoms and $\alpha_{c}=\left.\left(d E_{c} / d x\right)\right|_{\Delta x=0}$. For the conduction band, $m_{c}$ and $a_{c}$ are related to the electron. For the valence band, these values correspond to the heavy hole. The $E_{0}$ value characterizes also the exciton line broadening if the exciton is localized at composition fluctuations. In this case, $m_{c}$ is to be replaced by $m$, and $a_{c}$ by $a_{g}=d E_{g} / d x$. The smooth band tail (3) drastically changes under the action of exchange interaction (1). According to Karpov and Tsidil'kovskii [19], it becomes non-monotonic and temperature-dependent. A characteristic energy $E(T)$ appears within the energy gap, which separates the fully saturated collective mode from the polaron one. For a wide temperature range, $E_{0}<<k_{B} T<<\mathrm{S} E_{l} \mathrm{~S}$, a kind of «gap» occurs within the band tail and transforms into that plotted in fig. 3 . The energies displaced in fig. 3 are estimated as

$$
\left|E_{1}\right| \approx|J S\langle n\rangle / 2, \quad| E_{f} \mid \approx E_{0} / 4\left(k T / E_{0}\right)^{2}
$$

where $\langle n\rangle$ is associated with the relative average concentration of the impurities $\langle x\rangle$ and $\langle n\rangle=\langle x\rangle / a^{-3}$. Here $a$ is the lattice constant.

The peak in the density of states located near $E \approx E_{l}$ has a specific temperature dependence [19]

$$
g_{p} \approx g_{0} \exp \left(-\left[T / T_{0}\right]^{1 / 3}\right)
$$

for the case of non-correlated disorder and

$$
g_{p} \approx g_{0} \exp \left(-\left[T / T_{0}^{\prime}\right]^{8 / 3}\right)
$$

for a strongly correlated disorder. The temperatures $T_{0}$ and $T_{0}$, are expressed through the exchange constant $J$ and show the rate of thermal decay of the spin polarization.

The physical source of the appearance of this gap is the existence of an autolocalization barrier $L$. The states having characteristic radii smaller than $L$ become unstable with respect to spin polarization. The thermal disorder in the spin subsystem in semimagnetic semiconductors makes the density of states strongly temperature dependent.
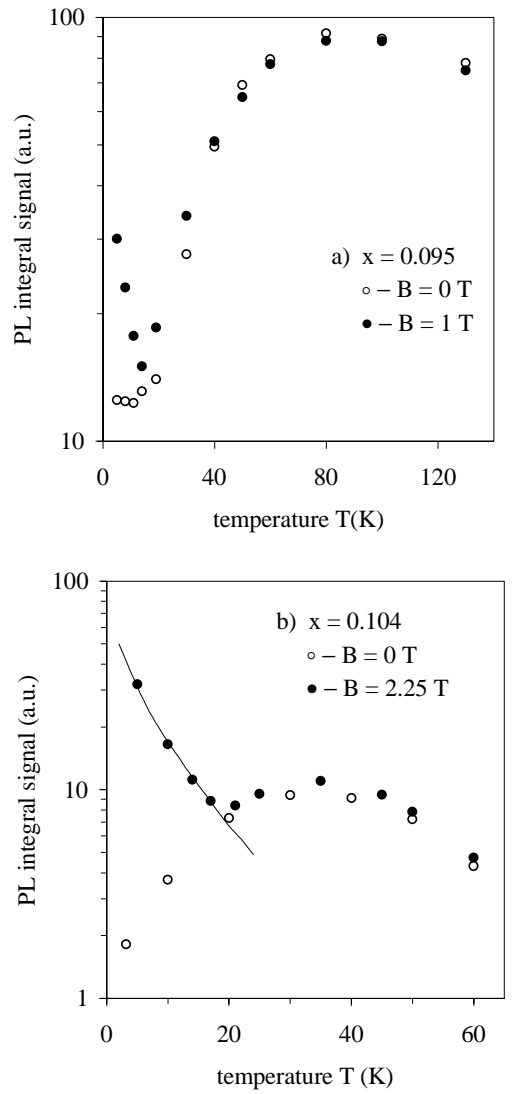

Fig. 4. Temperature dependencies of integral PL features with ( *) and without magnetic field (o) for $\mathrm{Hg}_{1-\mathrm{x}} \mathrm{Mn}_{\mathrm{x}} \mathrm{Te}$ of two compositions: (a) $x=0.095, B=B_{\text {max }}=1.1 \mathrm{~T}$; (b) $x=0.104, \quad B=B_{\max }=$ $=2.25 \mathrm{~T}$. Dashed curve in $(\mathrm{b})$ is the dependence calculated from eq. (12).

\section{Experimental results and discussion}

In this section, the behavior of the PL feature at different temperatures and magnetic fields is discussed. Fig. 4 shows the temperature dependence of the integral PL intensity for the same samples as displayed in fig. 1,2 with and without the magnetic field. To start the discussion, let us note that temperature-assisted non-monotonic growth of the PL magnitude is likely a common property observed also in $\mathrm{Hg}_{1-x} \mathrm{Cd}_{\mathrm{x}} \mathrm{Te}$ crystals [21]. An anomalous increase of the PL intensity, a change of the line shape, and a large blue shift of the spectrum with temperature have been reported. These experimental facts have been interpreted in terms of alloy disorder generating exponential band tails and the effect of carrier localization on the minority carrier lifetime. Here, we specially emphasize the peculiarities which are inherent only to the semimagnetic alloys: a non-monotonic behavior of peak intensity for low magnetic fields, and a drastic narrowing of the PL feature down to a FWHM of $2 \mathrm{meV}$, which is really surprising for narrow-gap semiconductors. Conclusive evidence of at least two different mechanisms responsible for the PL gain was revealed through the temperature dependence of the PL integral at $B_{\max }$. This latter conclusion is also supported by the temperature behavior of the PL peak 


\section{Yu. I. Masur: Strong effect of magnetic field on the edge luminescence line...}

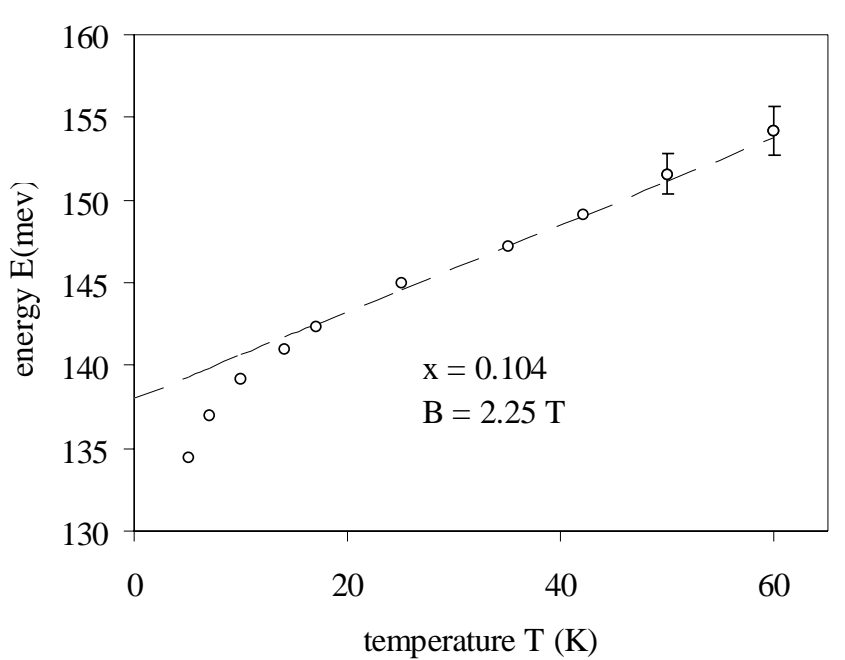

Fig. 5. Temperature behavior of the PL maximum position for $\mathrm{Hg}_{1-\mathrm{x}} \mathrm{Mn}_{\mathrm{x}} \mathrm{Te}\left(x=0.104, B=B_{\max }=2.25 \mathrm{~T}\right)$. Points - experiment, dashed line - the temperature dependence of $E_{g}$ calculated according to eq. (13). Bowing below $T=30 \mathrm{~K}$ reflects the excitonic corrections as well as the effect of exchange interaction between the spin of charge carriers and magnetic ions.

position $\left(K=K(B, T)=\hbar \omega_{\max }\right)$ plotted in fig. 5 . There is an explicit correlation between the bowing of $K(B, T)$ dependence, observed at low temperatures $(T<30 \mathrm{~K})$ in fig. 5, and the minimum for the integral PL intensity versus temperature in fig. 4 for the sample $x=0.104$ at $B_{\max }$.

In order to describe the PL behavior in the magnetic field, a modified Pidgeon-Brown approximation is used in the form described in Section 3. We assume the Luttinger parameters $\gamma_{1}=3, \tilde{\gamma}$ and $k=-1.65$ to be the same as for narrow-gap $\mathrm{Hg}_{1-\mathrm{x}} \mathrm{Mn}_{\mathrm{x}}$ Te alloys [22]. Due to the fact that the values of exchange interaction parameters determined by different experimental techniques $\left(-0.6 \leq N_{0} \alpha \leq-0.4 \mathrm{eV}\right.$, $0.6 \leq N_{0} \beta \leq 1.5 \mathrm{eV}$ ) are strongly scattered, we have chosen those obtained from similar experiments. It was reasonable to put first $N_{0} \beta=0.9$ and $N_{0} \alpha=-0.45$, following reference [22]. The fit of $\left.\mathrm{K}(\mathrm{B}, \mathrm{T})\right|_{T=5 K}$ for the high $B$ field limit has shown that the values of the exchange interaction parameters chosen are well adjusted within the reliability interval $( \pm 0.02 \mathrm{eV})$.

In our case of a comparatively high $\mathrm{Mn}^{2+}$ concentration, the possibility of manganese clusters also has to be taken into account. It leads to a modification of the $\hat{S}_{j}$ spin operator (eq. (1)). Being replaced by its thermal average $\left\langle\hat{S}_{J}\right\rangle$ in the molecular field approximation, it is determined by the magnetization of semimagnetics. For $\vec{B} \| z$-axis $\left\langle\hat{S}_{j}\right\rangle=\left\langle S_{z}\right\rangle$, and it becomes [23]

$\left\langle S_{z}\right\rangle=-S_{0} B_{5 / 2}\left(\frac{g_{M n} \mu_{B} B}{k_{B}\left(T+T_{0}\right)}\right)$

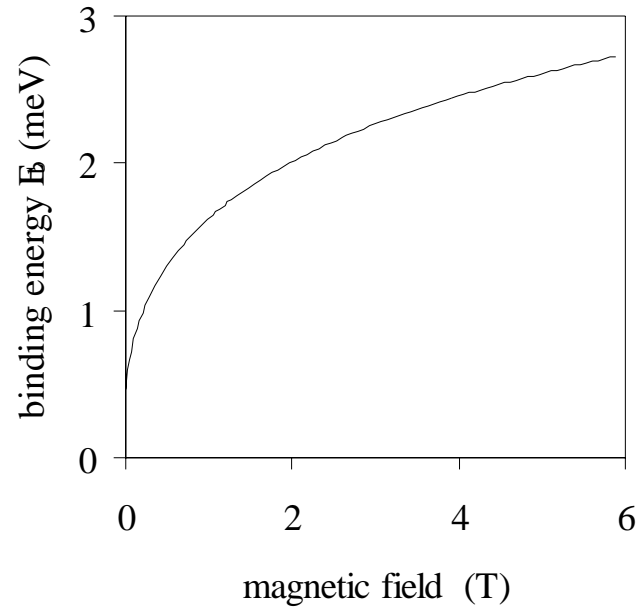

Fig. 6. Exciton binding energy for the ground state, calculated by eq. (2).

where $B_{5 / 2}$ is the Brillouin function for the spin $5 / 2$ and $g_{M n}$ is the gyromagnetic factor of the manganese ion, which is assumed to be 2 . Due to the antiferromagnetic interaction between the localized magnetic moments, a particular spin may respond to the external magnetic field with an effective temperature $T_{0}$ exceeding $T$, or it may be incorporated into a bound cluster $\left(S_{0}\right.$-factor). The effective temperature $T_{0}$ depends on $T$ and the manganese concentration. The $S_{0}$ values can be calculated as the probability of various magnetic clusters generated under the condition of random distribution of $\mathrm{Mn}^{2+}$ ions. For our case of $0.09<\mathrm{x}<0.11, S_{0}$ varies closely to 1 , and $T_{0}$ is between 8 and $10 \mathrm{~K}$. Under these circumstances, the renormalized effective $g$-factor for the conduction band (G6),

$g^{*}=g_{0} *+N_{0} \alpha\left\langle S_{z}\right\rangle /\left(\mu_{B} B\right)$,

becomes negative. Calculations with adjusted parameters show the following rearrangement of the energy gap in the magnetic field: energy levels $E_{a}(-1)$ and $E_{b}(-1)$ go up with the magnetic field increase, and it becomes $E_{b}(-1)>$ $>E_{a}(-1)$. This means a reduction of the energy gap $E_{g}$ when $\mathrm{B}$ grows due to the exchange interaction of heavy holes with the $\mathrm{Mn}^{2+}$ ions. The spin sublevels of the conduction band become higher and $E_{b}(0)>E_{a}(0)$. The spin splitting of the lowest Landau sublevels for the conduction band is small enough due to the small amplitude of $g^{*}$ of about 5. According to the selection rules for interband transitions, one can find that for $\vec{E} \mid \vec{B}$ the transition from the heavy hole band to the conduction band corresponds to the dominant sequence

$a_{\Gamma_{6}}(n-1) \rightarrow b_{\Gamma_{8}}(n), \quad n \geq 0$.

These transitions are of highest probability. Taking into account the thermal occupation of states at low temperatures, we suggest that the interband transition $b_{\Gamma_{8}}(0) \rightarrow a_{\Gamma_{6}}(-1) \quad$ with photon energy $\hbar \omega=E_{b}(0)-E_{a}(-1)=\Delta E$ has also to be included in the con- 


\section{Yu. I. Masur: Strong effect of magnetic field on the edge luminescence line...}

sideration. The dependence $\Delta E(B)$ calculated for this transition follows the $\left.\mathrm{K}(\mathrm{B}, \mathrm{T})\right|_{\mathrm{T}=5 \mathrm{~K}}$ dependence as shown in fig. 1 (inserts) and displays $\partial(\Delta E) / \partial B=0.4 \mathrm{meV} / \mathrm{T}$.

Exciton corrections have been included. The binding energy of the free exciton in the magnetic field is calculated with the help of eq. (2) and plotted in fig. 6. For the parameters chosen (effective masses for electron and hole are 0.01 and 0.5 respectively, dielectric constant $\varepsilon_{0}=17$ ) we find the value $E_{b i n}=0.4 \mathrm{meV}$ in the absence of the magnetic field and $E_{\text {bin }}=2.2 \mathrm{meV}$ when $B$ reaches $2.25 \mathrm{~T}$. The energy of the $b_{\Gamma_{8}}(0) \rightarrow a_{\Gamma_{6}}(-1)$ transition with excitonic correction is shown in fig. 1 (inserts) with a dashed line. One can easily observe a good quantitative agreement between the experimental data and theoretical results for the $\left.\mathrm{K}(\mathrm{B}, \mathrm{T})\right|_{\mathrm{T}=5 \mathrm{~K}}$ dependencies.

By compiling all the data presented above, we are able to put forward a model for their interpretation. The PL as a function of the temperature and magnetic field contains in fact two contributions: of the free exciton and plasma-like transitions. At a temperature of $T=5 \mathrm{~K}$ and without the magnetic field, observation of a distinct Wannier-Mott exciton contribution is not probable due to a small binding energy $\left(E_{b i n}=0.4 \mathrm{meV}\right)$. The temperature $(k T \approx 0.4 \mathrm{meV})$, potential fluctuations, free carrier screening and Stark broadening induced by the random field or strains result in a decay of the bound state. However, at the same temperature and the magnetic field increased up to $2 \mathrm{~T}$, the binding energy $(\sim 2 \mathrm{meV})$ is already sufficient to prevent the thermal destruction of an exciton. The band tail modification which occurs in diluted semiconductors and is described in Section 3 denotes in fact that the exchange interaction in the polaronic mode rectifies the forbidden band of semiconductors, and a «gap» arises in it. For $\mathrm{Hg}_{1-\mathrm{x}} \mathrm{Mn}_{\mathrm{x}} \mathrm{Te}$, this gap starts from the value $\left|E_{f}\right|$ (Eq.(5)), determined by the smearing parameter $E_{0}$ (eq. (4)) and the temperature $T$ strongly exceeding $E_{0} / k\left(k T>>E_{0}\right)$. According to our estimates, which are consistent with those in ref. [20], for the conduction band $E_{0}$ is of the order of $0.1 \mathrm{meV}$. Then $\left|E_{f}\right|$ $\sim 0.3 \mathrm{meV}$, and the latter means that the forbidden gap is clear from the tails produced by Gaussian fluctuations below $\left|E_{f}\right|$. The excitonic ground state falls within this energy region and becomes stabilized by the magnetic field, and thus produces a sharp resonance in PL (fig. 1). Therefore, we observe a narrow excitonic line. Its magnitude grows due to the enlargement of the excitonic oscillator strength and stabilization with respect to temperature. Vice versa, it begins to create the conditions under which the states of large radii have to be destroyed inevitably. Following [7], it can be shown that due to the quantization of electron motion in the plane perpendicular to $B$, the probability of being trapped into a fluctuation well grows in a strong magnetic field. The characteristic dimension of fluctuation well decreases with increasing $B$ as

$$
l_{B}=\left[\left(\frac{\hbar^{2} L}{\alpha m_{c}}\right)^{2} \frac{N}{x(1-x)}\right]^{1 / 3}
$$

where $L$ is the magnetic length, $L=(c \hbar / e B)^{1 / 2}$. The longi-

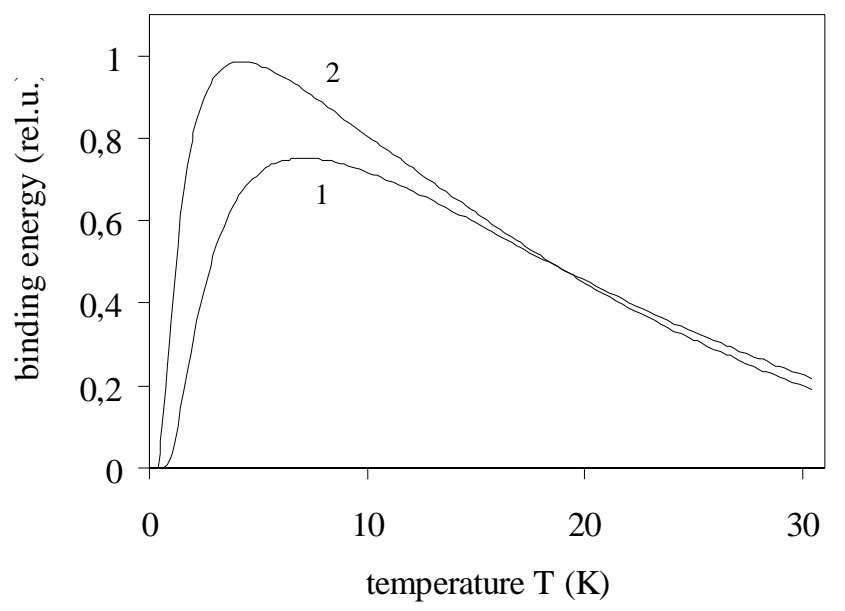

Fig. 7. Hole related FMP binding energy as a function of temperature for two compositions and applied magnetic field: curve $1-$ $x=0.0 .95, B=1.1 \mathrm{~T} ;$ curve $2-\mathrm{x}=0.104, B=2.25 \mathrm{~T}$.

tudinal exciton dimension, as follows from eq. (2), changes according to

$a_{B}^{-1}=\sqrt{\frac{2 \mu E_{b i n}}{\hbar^{2}}}$

When the magnetic field is increased, the ratio $\mathrm{h}=l_{B} / a_{B}$ becomes equal to $\ln B / B^{1 / 3}$. Whence $a_{B}$ approaches the value $l_{B}$, h comes close to unit. Thus, the electron is predominantly trapped by potential fluctuations. Here, the criterion of the critical field is fulfilled when $B=B=3.5 \mathrm{~T}$. This agrees well with the experimental data (fig. 1 ).

It should be mentioned that the magnetic field also affects the band tails of semimagnetics. By aligning both the spins of charge carriers and the magnetic moments of ions, it destroyes the self-consistent states in the spin-spin interacting subsystems. Preventing formation of the polaronic mode, in such a way the magnetic field renormalizes only the density of states $g(E)$, and the «gap» disappears when the semimagnetic becomes affected by a strong magnetic field [19]. For an intermediate magnetic field, the spin-spin interacting subsystem is controlled by a rigorous competition between the magnetic field and temperature. According to our calculations, the dependence of free energy $F(B, T)$ which determines the thermodynamic properties of the subsystem of charge carriers and magnetic ions is a strongly nonmonotonic function of temperature [24]. Fig. 7 shows the magnetic polaron binding energy for the hole-magnetic ions spin-spin interaction in $\mathrm{Hg}_{1-\mathrm{x}} \mathrm{Mn}_{\mathrm{x}}$ Te crystals under investigation. It can be seen that the magnetic field $B$ destroys bound polaronic states when the temperature is low $(T<1.5 \mathrm{~K})$. However, as the temperature approaches the range $4 \leq T \leq 6 \mathrm{~K}$, the magnetic field effect is to a great extent eliminated. Rising temperature at a constant magnetic field $B$ leads to a suppression of the polaronic mode. Therefore, in every single particular case it is possible to assess the most favorable conditions for observation of the polaron effect and the band tail «gap» [19]. 


\section{Yu. I. Masur: Strong effect of magnetic field on the edge luminescence line...}

We have to underline that the findings reported are in agreement with an excitonic mechanism. Furthermore, the temperature-enhanced change of the PL spectra is also consistent with this concept. Fig. 3 demonstrates the abrupt temperature quenching of the narrow PL feature and subsequent slow flaring-up of a broad plasma-like structure. According to the model proposed, the strong temperature quenching and broadening is caused by thermal dissociation of the exciton. At $T=20 \mathrm{~K}$ the exciton binding energy becomes equal to the thermal energy $k T$. Moreover, the excitonic ground state merges into the region with an increased density of states, which descends (in accordance with eq. (5)) quadratically with temperature. The excitonic state now becomes quenched. Further, the temperature induced bandto-band photoluminescence is the dominant feature when the temperature exceeds $T=35 \mathrm{~K}$. The $K(B, T)$ dependence evolves into the function of temperature only and strictly follows the temperature dependence of $E_{g}$.

Up to this point, we have analyzed the conditions which would be favorable for exciton formation mainly from the conduction band. However, there are circumstances facilitating free exciton formation from the side of the valence band. One can see from fig. 6 that the hole-related FMP is equally possible within the temperature and magnetic field ranges of visible exciton existence. The temperature behavior of the PL peak at $B$ equal to $B$ (fig. 5) shows an abrupt deflection from the linear law which is proper for interband transitions. This deflection can be related to formation of a hybrid complex of a free exciton trapped by a hole-related free magnetic polaron. This interaction also tends to strengthen the discrete electronic state. So, in fact we have here all conditions for successful observation of a magnetically trapped exciton in narrow-gap semimagnetic semiconductors.

According to [19], emission dominated by the charge carriers localized at the density of states peaks $E \sim E_{1}$ (eq. (5)) can be observed as well. This conclusion means that the emission spectra of semimagnetics should display peaks at the energies close to $E_{l} . E_{l}$ peculiarities of $g(E)$ distribution should be observed for a wide temperature range $E_{0}<<k T<<\left|E_{1}\right|$ ( for our case $\left|E_{1}\right|>>112 \mathrm{meV}$ ). We do not rule out that a comparatively wide temperature induced emission observed in our case can be related to the abovementioned peculiarities of the band tail in semimagnetics. It is also interesting to note that the temperature-dependent behavior of PL intensity is described by the law

$\ln (I)=\ln \left(I_{0}\right)-A T^{1 / 3}$

where $A$ is a fitting parameter. The dependence presented by eq. (12) is plotted in fig. 2 with a dashed line. The law presented by eq. (12) immediately arises from eq. (3) if one assumes that the band tail is pinned to the conduction band. The energy gap for our compounds follows the linear dependence

$E_{g}(\mathrm{~T})=E_{0}+5.35 \cdot 10^{-4}(1-4.9 \cdot \mathrm{x}) \cdot T$.

However, $g(E, T)$ has the same temperature dependence given by eq. (6) and related to exchange interactions in semimagnetics. Concerning the state of the hole within a free excitonic complex, one can admit, taking into account the evidence of the free polaron, that the hole state imposes its temperature dependence on the intensity of excitonic photoluminescence, especially when this state is located in close proximity of $E_{l}$ peculiarity. In any case, the possibility of manifestation of the $E_{1}$ mechanism in the PL observed in our case cannot be ruled out and requires a special consideration elsewhere.

\section{Conclusion}

A strongly pronounced dependence of the PL signal in diluted narrow-gap magnetic semiconductors $\mathrm{Hg}_{1-\mathrm{x}} \mathrm{Mn}_{\mathrm{x}} \mathrm{Te}$ on the magnetic field and temperature reflects a complicated interplay between the exchange interaction and energy bands rearrangement, fluctuonic processes and disorder. At every particular stage, the most favorable conditions occur to manifest the numerous aspects of this interplay and substantially affect the PL intensity. A really unique chance to observe a sharp excitonic resonance in narrow-gap semimagnetics was demonstrated here for $\mathrm{Hg}_{1-\mathrm{x}} \mathrm{Mn}_{\mathrm{x}} \mathrm{Te}$ with compositions $0.9<$ $<x<0.11$. These materials have a positive energy gap $\left(E_{g}>\right.$ $>100 \mathrm{meV})$ and a negative $g$-factor $(|g *| \approx 5)$ for the conduction band. Due to a specific compromise between the magnetic field and temperature, a gap appears within the forbidden band of semiconductors. It becomes strongly purified with respect to the band tails generated by Gaussian fluctuations in semimagnetics. Being fully controlled by exchange interactions, this gap is temperature, magnetic field, and composition dependent. Its role here is widely discussed. We state that $B$-related strengthening of exciton binding leads to a possibility of the excitonic ground state dropping within the «tailless» forbidden band. This discrete state is mainly responsible for the PL flare-up in the magnetic field. Starting from this, we are able to explain self-consistently the peculiarities of PL manifestation in narrow-gap $\mathrm{Hg}_{1-\mathrm{x}} \mathrm{Mn}_{\mathrm{x}} \mathrm{Te}$ depending on both the temperature and the magnetic field. At a low temperature $(T>5 \mathrm{~K})$ and a negligible magnetic field, a long-living exciton state does not arise due to a low binding energy and a high probability of incoherent trapping of charge carriers by fluctuations in narrow-gap materials. The magnetic field of an intermediate strength facilitates the binding of Coulomb-interacting charge carriers into an exciton. Entering the tailless gap, this discrete state of a large radius gives rise to a sharp PL feature achieving its maximum at $1 T<B<3 T$ for the composition under investigation. For a larger magnitude of the magnetic field $(B>$ $>4 T$ ), the excitonic state undergoes an intensive decay caused by electron trapping in the magnetically modified band tail, which arises again for the suppression of the fluctuonic mode by the magnetic field in the ultra-quantum limit. Then, the PL signal is controlled mainly by interband transitions with a subsequent temperature-assisted flaring up. The experimental data available and theoretical estimates show also the possibility of optical manifestation of a holerelated FMP. It strongly affects the hole kinetics and, when included in an exciton, significantly stabilizes the complex. The model of a magnetically trapped exciton can describe well the PL behavior observed. Thus, a novel concept pre- 


\section{Yu. I. Masur: Strong effect of magnetic field on the edge luminescence line...}

sented in this paper substantially enriches the physical picture of processes developing under the control of magnetic field and temperature in spin-spin interacting systems. Studies of PL gave new evidence that discrete hybrid excitation exists even in the case of narrow-gap semimagnetics. We are aware that there are still some uncertanities in the analysis. To clarify them, more investigations should be performed at the temperature $T<5 \mathrm{~K}$ and with a picosecond optical technique used to explore the excitonic kinetics.

\section{Acknowledgments}

The author would like to acknowledge Prof. G. G. Tarasov and Dr. J. W. Tomm for a number of helpful and illuminating discussions, and Dr. O. A. Bodnaruk for supplying highquality $\mathrm{Hg}_{1-\mathrm{x}} \mathrm{Mn}_{\mathrm{x}}$ Te single crystals.

\section{References}

1. B. I. Shklovskii and A. L. Efros, Electronic Properties of Doped Semiconductors, Springer-Verlag, Berlin (1984).

2. J. Gebhardt, G. Nimtz, B. Schlicht, and J. Stadler, Phys. Rev. B 32, p. 5449 (1985).

3. M. Shayegan, H. D. Drew, D. A. Nelson, and P. M. Tedrov, Phys. Rev. B 31, p. 6123 (1985).

4. A. Mycielski and J. Mycielski, J. Phys. Soc. Japan, Suppl. A, 49, p. 807 (1980).

5. J. A. Gay and A. Golnik, Acta Phys.Polon. A 67, p. 307 (1985).
6. P. Wolff and J. Warnock, J.Appl.Phys. 55, p. 2300 (1984).

7. V. I. Ivanov-Omskii, S. I. Kochanovskii, R. P. Seysyan, V. A. Smirnov, V. A.Yukish, S. U. Yuldashev, and Al. L. Efros, Solid State Commun., 46, p. 25 (1983).

8. B. L. Gel'mont, R. R. Galazka, E. M. Vakhabova, V. I. Ivanov-Omskii, I. T. Postolaki, and V. A. Smirnov, Fiz.Tech.Poluprovodn, 20, p. 73 (1986), [Sov.Phys. 20, p. 42 (1986)].

9. J. Warnock, R. N. Kershaw, D. Ringley, K. Dwight, A. Wold, and R. R. Galazka, Solid State Commun. 54, p. 215 (1985).

10. A. Golnik, J. Ginter, and J. Gay, J.Phys. C 16, p. 6083 (1983).

11. Yu. I. Mazur, G. G. Tarasov, V. Jahnke and J. W. Tomm, Infrared Phys. and Technol. 36, p. 929 (1995).

12. J. Mycielski in: Semiconductors and Semimetals v. 25 (R.K.Willardson and A.C.Beer, Eds) Academic Press (1988) 311-344.

13. W. Leung and L. Liu, Phys.Rev. B 8, p. 3811 (1973).

14. R. R. Galazka and J. Kossut, Lecture Notes in Physics ( SpringerVerlag) 133, p. 245 (1984).

15. W. Ekardt, Solid State Commun., 16, p. 233 (1975).

16. Y. Yafet, R. W. Keyes, and E. N. Adams, J.Phys.Chem.Solids, 1, p. 137 (1956).

17. M. von Ortenberg, J.Phys.Chem.Solids, 34, p. 397 (1973).

18. B. A. Aronzon and I. M. Tsidilkovskii, Phys.Stat.Sol.,(b) 157, p. 17 (1990).

19. V. G. Karpov and E. I. Tsidil'kovskii, Phys.Rev., B 49, p. 4539 (1994).

20. S. D. Baranovskii and A. L. Efros, Fiz.Tech.Poluprovodn.Semicond., 11, p. 2233 (1978), (Sov.Phys.Semicond., 12, p. 1328 (1978)).

21. F. Fuchs and P. Koidl, Semicond.Sci.Technol., 6, p. 71 (1991).

22. G. Bastard, C. Rigaux, Y. Gulder, A. Mycielski, J. K. Furdina, and D. P. Mullin, Phys.Rev., B 24, p. 1961 (1981).

23. N. B. Brandt and V. V. Moshchalkov, in: Advances in Physics, Ed. D. Sherrington (Taylor and Francis Ltd, London, UK) (1984) 246.

24. G. G. Tarasov, Yu. I. Mazur, A. S. Rakitin, and S. R. Lavoric, Proc. $S P I E$, v. 3182, p. 282 (1997).

\section{СИЛЬНИЙ ВПЛИВ МАГНІТНОГО ПОЛЯ НА ШИРИНУ ЛІНЇ̈ ГРАНИЧНОЇ ЛЮМІНЕСЦЕНЦІї В РОЗБАВЛЕНИХ МАГНІТНИХ ВУЗЬКОЗОННИХ НАПІВПРОВІДНИКАХ $\mathrm{Hg}_{1-\mathrm{X}} \mathrm{Mn}_{\mathrm{x}} \mathrm{Te}$}

\section{Ю. I. Мазур}

\section{Інститут фізики напівпровідників НАН Украӥни}

Резюме. Досліджено особливості фотолюмінесценції у вузькозонних твердих розчинах $\mathrm{Hg}_{1-\mathrm{x}} \mathrm{Mn}_{\mathrm{x}} \mathrm{Te}(0.09<x<0.11)$ у випадку комбінованого впливу магнітного поля та температури. Виявлено сильне резонансне підсилення крайової люмінесценції та звуження ширини іiї лінії до значень $<2.5 \mathrm{meV}$. Спостережувана поведінка фотолюмінесценції інтерпретується ,в рамках моделі магніто-захопленого екситона.

\section{СИЛЬНОЕ ВЛИЯНИЕ МАГНИТНОГО ПОЛЯ НА ШИРИНУ ЛИНИИ КРАЕВОЙ ЛЮМИНЕСЦЕНЦИИ В РАЗБАВЛЕННЫХ МАГНИТНЫХ УЗКОЗОННЫХ ПОЛУПРОВОДНИКАХ $\mathrm{Hg}_{1-\mathrm{X}} \mathrm{Mn}_{\mathrm{x}} \mathrm{Te}$}

\section{Ю. И. Мазур}

\section{Институт физики полупроводников НАН Украины}

Резюме. Исследованы особенности фотолюминесценции в узкозонных твердых растворах $\mathrm{Hg}_{1-\mathrm{X}} \mathrm{Mn}_{\mathrm{x}} \mathrm{Te}(0.09<x<0.11)$ при комбинированном воздействии магнитного поля и температуры. Обнаружено сильное резонансное усиление краевой люминесценции и сужение ширины ее линии до значений $<2.5 \mathrm{meV}$. Наблюдаемое поведение фотолюминесценции интерпретируется в рамках модели магнито-захваченного экситона. 